\title{
Response
}

\section{Age at Diagnosis and the Risk of Diabetic Nephropathy in Young Patients with Type 1 Diabetes Mellitus (Diabetes Metab J 2021;45:46-54)}

\author{
Jong Ha Baek ${ }^{1}$, Jae Hyeon Kim² \\ ${ }^{1}$ Department of Internal Medicine, Gyeongsang National University Changwon Hospital, Gyeongsang National University College of Medicine, Changwon, \\ ${ }^{2}$ Department of Medicine, Samsung Medical Center, Sungkyunkwan University School of Medicine, Seoul, Korea
}

We appreciate Professor Yang and Sohn's interest in and comments on our article, entitled "Age at diagnosis and the risk of diabetic nephropathy in young patients with type 1 diabetes mellitus," which was published in the Diabetes and Metabolism Journal [1].

Due to the limitations of our cross-sectional study design, it was difficult to interpret the potential causality of the inverse association between disease duration and estimated glomerular filtration ratio (eGFR) according to age at diagnosis. As professor Yang and Sohn's mentions, other prospective and longitudinal studies are needed to elucidate the causal relationship between age at diagnosis and reductions in eGFR in the future.

It is important to consider whether angiotensin receptor blocker (ARB)/angiotensin converting enzyme inhibitor (ACEi) class drugs were used to prevent the progression of albuminuria or chronic kidney disease. Meanwhile, in this study, the proportion of antihypertensive drug use in the younger age group ( $<20$ years) was numerically higher than in other age groups ( $32.2 \%$ vs. $22.4 \%$ vs. $24.1 \%$ ). In addition, the use of anti-hypertensive drugs was not associated with risk of diabetic nephropathy in this study (odds ratio, 1.09; 95\% confidence interval, 0.93 to 1.24). However, as you mentioned, the use of ACEi/ARB class drugs is an important therapeutic strategy to prevent the progression of diabetic nephropathy especially in a young adult population.
Not only chronic hyperglycemia but also glycemic variability is associated with diabetic complications. Even though glycosylated hemoglobin levels were adjusted in this study, we were not able to evaluate glycemic variability directly. Instead, we speculated that more intensive insulin therapy requiring higher doses of insulin in a young age group $(<20$ years) may reflect higher glycemic variability.

In this study, when C-peptide levels were categorized by quartile, eGFR (mean $84.5 \mathrm{~mL} / \mathrm{min} / 1.73 \mathrm{~m}^{2}$ vs. $99.5 \mathrm{~mL} /$ $\mathrm{min} / 1.73 \mathrm{~m}^{2}$ vs. $94.6 \mathrm{~mL} / \mathrm{min} / 1.73 \mathrm{~m}^{2}$ vs. $90.7 \mathrm{~mL} / \mathrm{min} / 1.73 \mathrm{~m}^{2}$ from lowest to highest quartile; $P=0.546$ ) and urine albumincreatinine ratio (median $16.7 \mathrm{mg} / \mathrm{g}$ vs. $9.8 \mathrm{mg} / \mathrm{g}$ vs. $9.7 \mathrm{mg} / \mathrm{g}$ vs. $11.8 \mathrm{mg} / \mathrm{g}$ from lowest to highest quartile) were comparable between C-peptide quartiles. We could not find an association between C-peptide levels and diabetic complications.

There was relatively more heterogeneity in the classification of diabetes in adult-onset diabetes rather than childhood-onset diabetes [2]. In this study, the baseline characteristics of the study population were assessed after a median 14 years of disease duration and the possibility that cases with positive autoantibodies at diagnosis were converted to negative status over time cannot be ruled out $[3,4]$. However, as professor Sohn mentioned, a more detailed diagnostic approach to rule out other types of diabetes (e.g., monogenic diabetes) should be considered.

Eligibility for this study included those who were registered
Corresponding author: Jae Hyeon Kim (D) https://orcid.org/0000-0001-5001-963X Department of Medicine, Samsung Medical Center, Sungkyunkwan University School of Medicine, 81 Irwon-ro, Gangnam-gu, Seoul 06351, Korea

E-mail: jaehyeon@skku.edu
This is an Open Access article distributed under the terms of the Creative Commons Attribution Non-Commercial License (https://creativecommons.org/licenses/by-nc/4.0/) which permits unrestricted non-commercial use, distribution, and reproduction in any medium, provided the original work is properly cited. 
in the Korea National Health Insurance Service (KNHIS) program for reimbursement. Due to the retrospective nature of the study, patients were enrolled if they met at least one of the reimbursement criteria and history of diabetic ketoacidosis was checked through patient recall. As a result, it was difficult to accurately determine if patients had a history of DKA after diagnosis of diabetes.

We would like to express our sincere gratitude to professor Yang and Sohn again for their valuable comments. We fully agree with professors' suggestion that a nationwide prospective registry for type 1 diabetes mellitus (T1DM) is needed to expand our understanding of the natural course of T1DM in Korea.

\section{CONFLICTS OF INTEREST}

No potential conflict of interest relevant to this article was reported.

\section{REFERENCES}

1. Baek JH, Lee WJ, Lee BW, Kim SK, Kim G, Jin SM, et al. Age at diagnosis and the risk of diabetic nephropathy in young patients with type 1 diabetes mellitus. Diabetes Metab J 2021;45:46-54.

2. Redondo MJ, Evans-Molina C, Steck AK, Atkinson MA, Sosenko J. The influence of type 2 diabetes-associated factors on type 1 diabetes. Diabetes Care 2019;42:1357-64.

3. Liu L, Li X, Xiang Y, Huang G, Lin J, Yang L, et al. Latent autoimmune diabetes in adults with low-titer GAD antibodies: similar disease progression with type 2 diabetes: a nationwide, multicenter prospective study (LADA China Study 3). Diabetes Care 2015;38:16-21.

4. Kawasaki E, Fukuyama T, Uchida A, Sagara Y, Tamai H, Nakano $\mathrm{Y}$, et al. Characterization of patients with diabetes who were incidentally found to be glutamic acid decarboxylase autoantibody-positive by bridging-type enzyme-linked immunosorbent assay. J Diabetes Investig 2020;11:1507-10. 